\title{
Magnetic ordering and interactions in iron-filled carbon foam
}

Filippo S. Boia ${ }^{a}$, , Xiaotian Zhanga, Omololu Odunmbakua, JiaChen Xiaa, Ayoub Taallaha and Mark Baxendale ${ }^{b *}$

${ }^{a}$ College of Physical Science and Technology Sichuan University, Chengdu China.


Email corresponding author: f.boi@scu.edu.cn Email second corresponding author: m.baxendale@qmul.ac.uk

\begin{abstract}
Ferromagnetically filled graphitic-carbon foams are a new class of materials characterized by an open-cell structure continuously filled with ferromagnetic crystals. Targetted design for applications requires knowledge of the magnetic ordering and the interaction between magnetic moments in the filling and those due to the spin of the delocalised $\pi$-electrons which contribute to thermal and electrical conduction in the graphitic carbon. Magnetometry together with electron spin resonance, thermoelectric, and calorimetric measurements on iron-filled carbon foam reveal ferromagnetic responses at room temperature with a transition to antiferromagnetic behaviour in some fraction of the filling at $130 \mathrm{~K}$. This observation is consistent with the $\alpha-\mathrm{Fe}(\mathrm{bcc})$ to $\gamma-\mathrm{Fe}(\mathrm{fcc})$ transition at this temperature predicted by others. Seebeck coefficient measurements revealed a behaviour typical of
\end{abstract}


unmodified graphitic carbon indicating the absence of any interaction between the magnetic moments of the filling and the conduction electrons in the graphitic carbon.

\section{Introduction}

Ferromagnetically filled carbon materials are multifunctional systems which can exploit controllable magnetic properties and important electromagnetic waves dissipation characteristics to be used in multiple technological applications. This class of materials frequently includes 1) graphene coated iron-filled nano-composites [1], 2) amorphous-carbon-coated iron/iron-oxide-filled composites [2-11] 3) ferromagnetically filled carbon foam [12-15], 4) ferromagnetically filled carbon nanotubes (Me@CNTs, where Me is the encapsulated metal or metal carbide) [16 28], 5) ferromagnetically filled carbon onions (Me@CNOs) [28] and 6) ferromagnetically filled CNTs-polymer composites [16, 29-35]. Typical examples of used polymers for films fabrication include (Ca-SA)/deacetylated konjac glucomannan (DKGM) [29], P(GMA-EGDMA) [34], Sodium alginate [32], P(GMA DVB) [35] and polyurethane-urea (Spandex) [16].

In typical iron-filled amorphous-carbon-coated or polymer-based composites the ferromagnetic/paramagnetic particles have been frequently coated with: 1) amorphous carbon [2-11] through the use of high intensity ultrasound for the decomposition of $\mathrm{Fe}(\mathrm{CO})_{5}$ and subsequent annealing or 2) graphene layers which have been reported to exhibit excellent microwave absorption performances [1]. Recent works have also shown that multishelled $\mathrm{ZnO}$ spheres could be considered possible candidates to encapsulate functional materials of interest [31]. 
The key advantages of ferromagnetically filled graphitic-carbon foams [12-15] relative to other, comparable systems are 1) the high-volume-fraction of the continuous ferromagnetic filling, 2) typically high saturation magnetizations comparable to those of bulk iron, and 3) the scalability of the production method, which involves fusion of $\mathrm{Fe}_{3} \mathrm{C}$ nanocrystals encapsulated by spherical graphitic carbon shells by annealing at high temperature [12-15]. The applications of this composite material exploit the controllable ferromagnetic properties or the inherent electromagnetic absorption dissipation properties of the magnetic moments in the filling and the conduction electrons in the graphitic carbon.

The nature of the magnetic ordering and the question of whether the magnetic moments interact with those due to the spin of the $\pi$-electrons in the graphitic carbon - which contribute to thermal and electrical conduction - is therefore important if structure-property relations are to be understood and tailored design is to be achieved.

Here we address these questions in a novel investigation where we focus our attention on the magnetometry together with electron spin resonance (ESR), thermoelectric, and calorimetric measurements properties of iron-filled magnetic carbon foam.

We observe ferromagnetic hysteresis with a saturation magnetization $\mathrm{Ms}=180$ emu/g and a coercivity $\mathrm{Hs}=29$ Oe at room temperature and a reduction in magnetisation in the zero-field-cooled (ZFC) characteristic at $130 \mathrm{~K}$. On the basis of the ESR and specific heat capacity measurements we conclude that this ZFC feature 
is the result of a transition from ferromagnetic to antiferromagetic ordering in some of the filling. Thermoelectric measurements indicate no interaction between the magnetic moments and the $\pi$-electron system.

\section{Experimental}

The iron-filled graphitic carbon foam was fabricated by using the two-stage process reported in Ref. 2-4. Self-organised $\mathrm{Fe}_{3} \mathrm{C}$ nanocrystals encapsulated by graphitic carbon shells were firstly produced by ferrocene pyrolysis at $900{ }^{\circ} \mathrm{C}$. Annealing was then performed in the same reactor at the temperature of $1000{ }^{\circ} \mathrm{C}$ under $\mathrm{Ar}$ flow.

The characterization was carried out with: a JSM-7500F 5-20 kV scanning electron microscope (SEM), an Empyrean PANalytical powder X-ray diffractometer (Cu $\mathrm{K}-\alpha, \lambda=0.154 \mathrm{~nm}$ ), and a $200 \mathrm{kV}$ American FEI Tecnai G2F20 transmission electron microscope (TEM). Magnetisation measurements were performed with a vibrating sample magnetometer (Quantum Design). ESR measurements were performed using a JEOL JES-FA200. Differential scanning calorimetry (DSC) measurements were performed with a TA DSC 2920 in the temperature range -181 $C$ to $130 \circ C$ with a Sapphire reference. Seebeck measurements were performed with an MMR technologies, Inc. SB-100 Seebeck measurement system and K-20 temperature controller.

\section{Results and Discussion}

The morphology of the powder extracted from the reactor is detailed in Fig.1. 


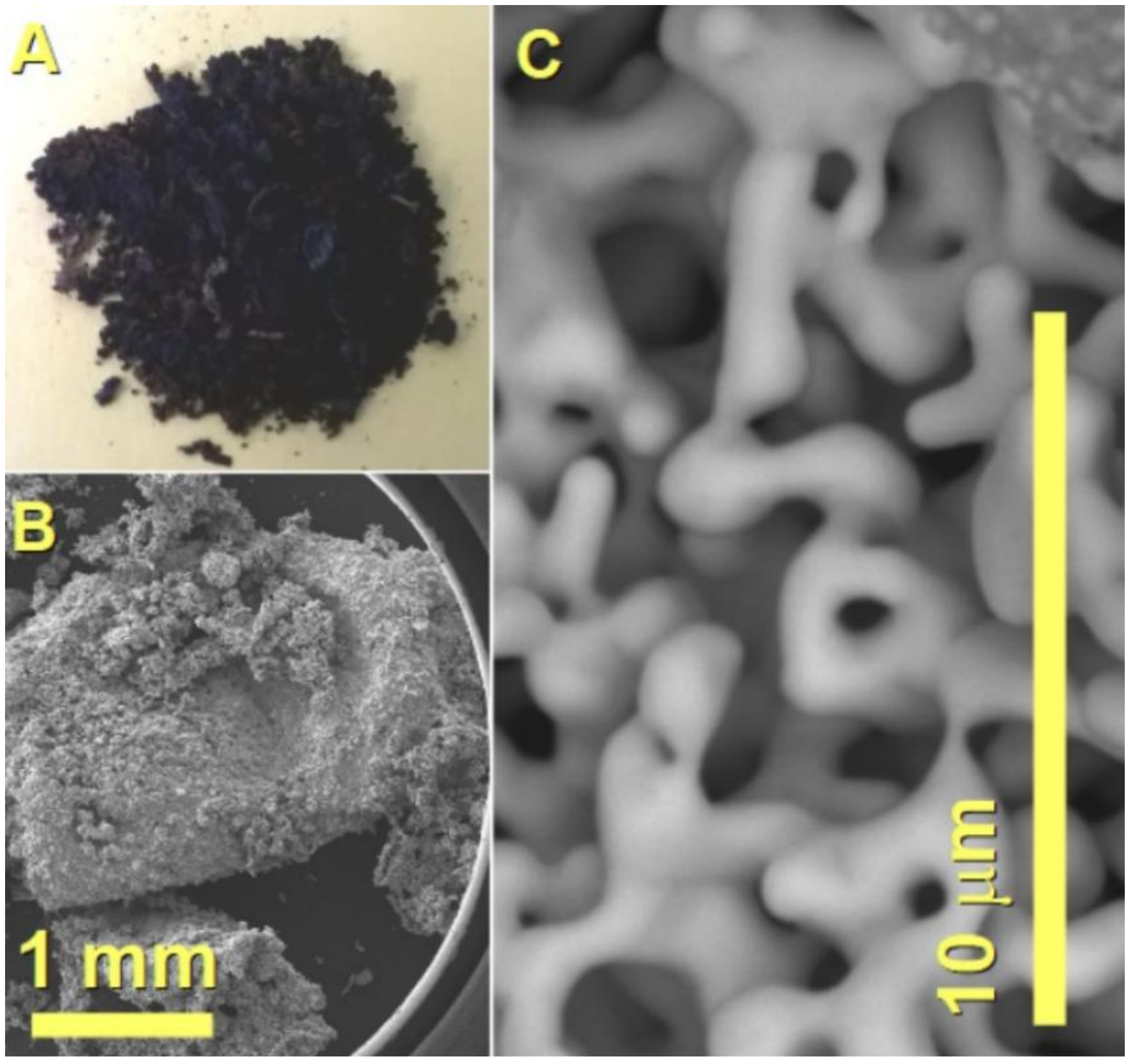

Figure 1: Photograph $(A)$ and SEM micrographs $(B, C)$ of iron-filled graphitic carbon

foam.

The SEM micrographs in Fig.1B,C show an interconnected network. The mass contrast observed in backscattered electron SEM images revealed a high volume fraction of the heavier iron, Fig.Supp.1. 
The XRD data in Fig.2 shows the progressive disappearance of $\mathrm{Fe}_{3} \mathrm{C}$ features as a consequence of annealing. 


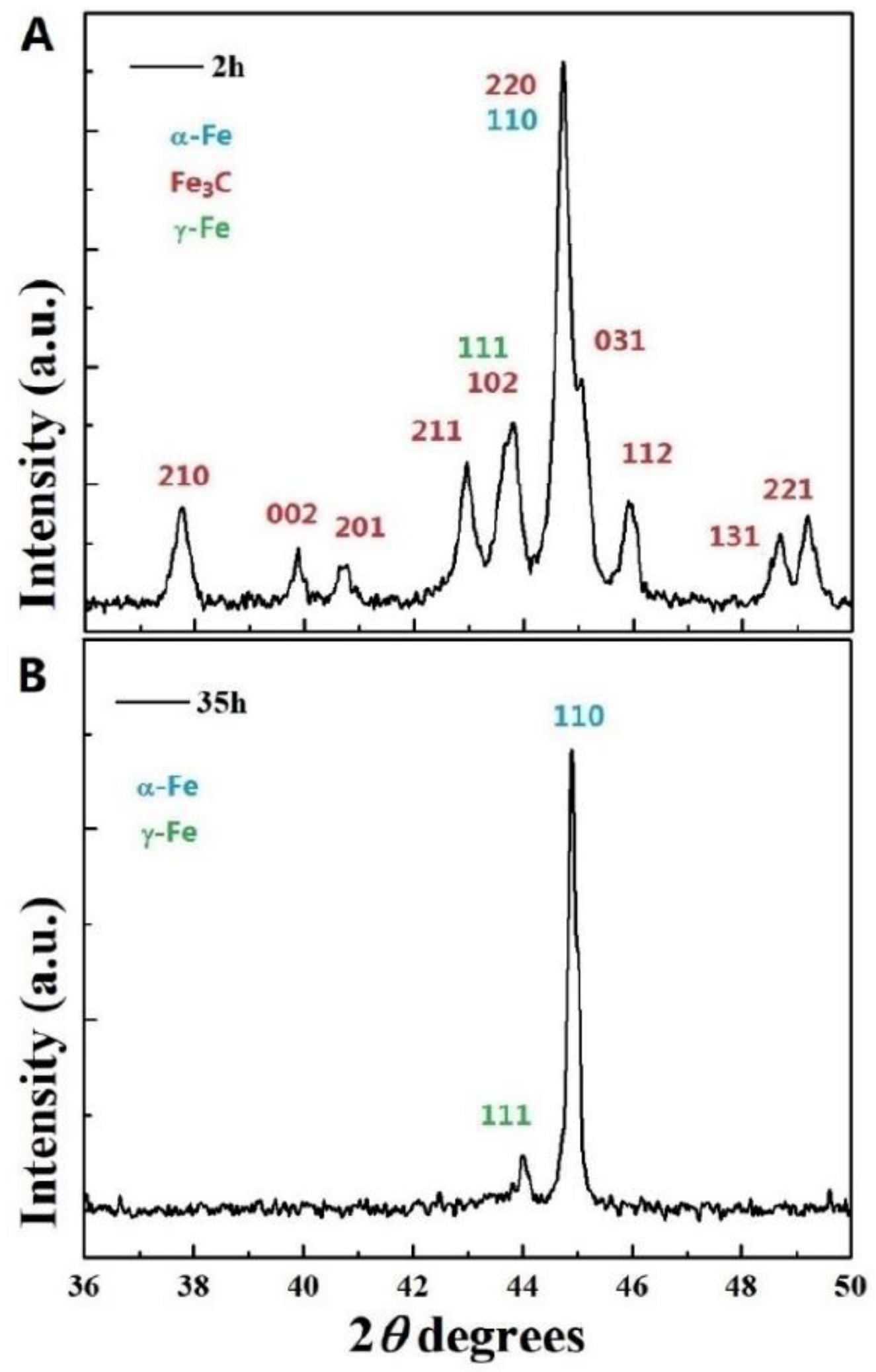

Figure 2: XRD analyses of the iron-filled graphitic carbon foam showing structural variation as a consequence of annealing for (A) $2 \mathrm{~h}$ and (B) $35 \mathrm{~h}$. 
The intense diffraction peak observed at $2 \theta \approx 45$ degrees is that of the 110 reflection of $\alpha$-Fe. The TEM micrograph in Fig.Supp.2 is that of a typical iron/graphitic-carbon interface. Raman spectroscopy revealed the $G$ and $D$ peaks with a sp3-rich arrangement, in agreement with ref. 13-15 (Fig.Supp.3).

On the Raman spectrum shown in Fig.Supp.3 two bands could be detected at approximately $\sim 1300 \mathrm{~cm}-1$ and $\sim 1600 \mathrm{~cm}-1$. The first band ( $D$ band) is associated to the disordered induced scattering produced by imperfections or loss of hexagonal symmetry in the carbon structure and or presence of sp3-defects. The second band ( $\mathrm{G}$ band) can instead be assigned to the Raman active $2 \mathrm{E}_{2 \mathrm{~g}}$ mode which is generally observed in graphite like materials [43-45]. These observations are in agreement with those reported in ref. [13-15].

The room temperature variation of magnetization with applied magnetic field, Fig.3A, revealed ferromagnetic hysteresis with saturation magnetisation $M_{\mathrm{s}}=180 \mathrm{emu} / \mathrm{g}$, comparable to that of bulk iron $(220 \mathrm{emu} / \mathrm{g})$, and a coercivity $H_{c}=29 \mathrm{Oe}$. However, the ZFC characteristic shows a depletion of magnetization greater than the trend at approximately 130 K, Fig.3B.

ESR analyses through $130 \mathrm{~K}$ revealed further evidence for a transition in magnetic ordering below $130 \mathrm{~K}$, Fig.4. The broad differential absorptions typical of ferromagnetic systems have minor superimposed features with g-factor values of 1.99 (attributable to the $\pi$-electron contribution [36,37]). 

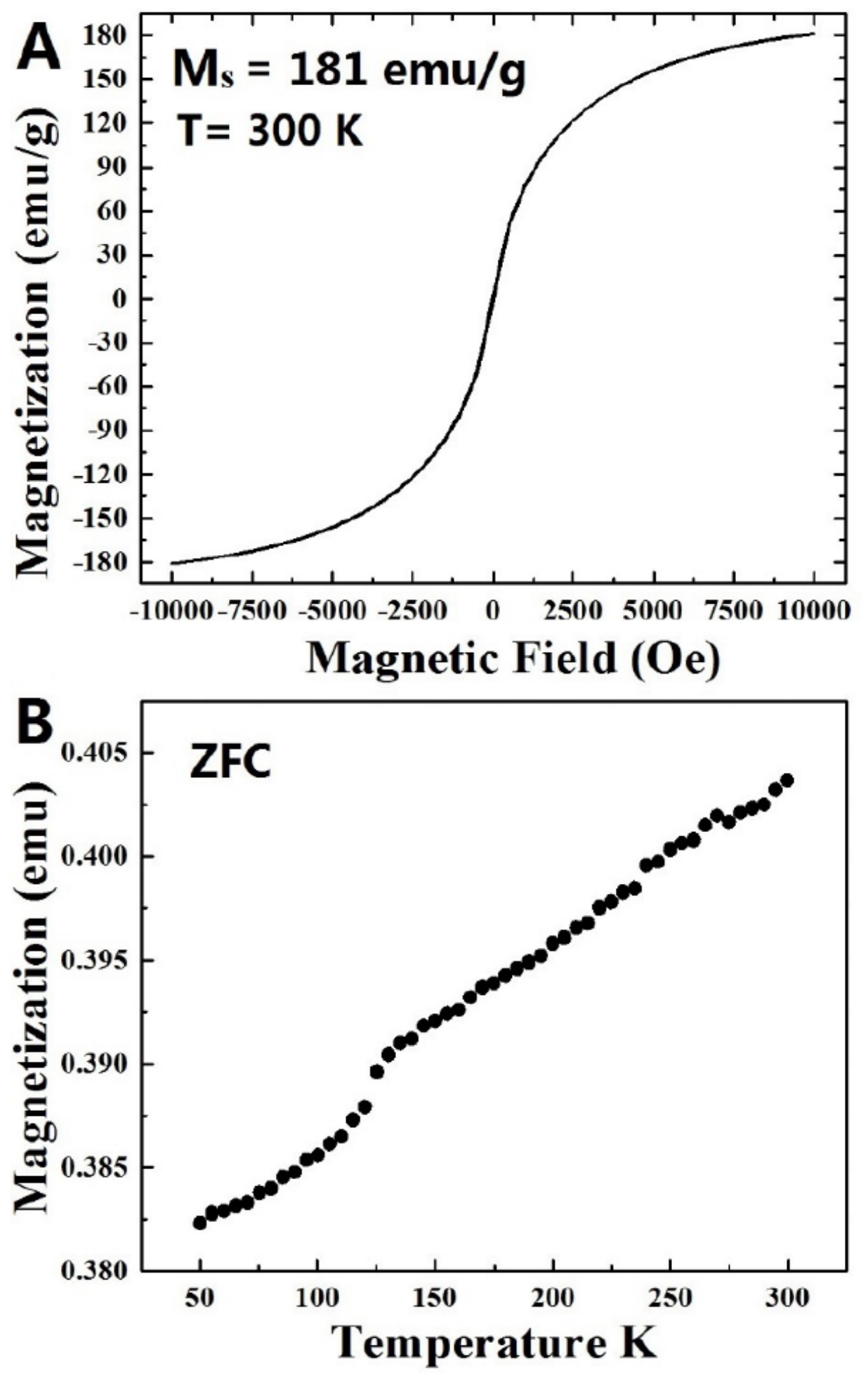

Figure 3: Magnetization versus (A) applied magnetic field strength at room temperature, and (B) temperature with no applied field (ZFC). 
However, at a temperature less than $130 \mathrm{~K}$ there is also a significant narrow feature with a g-value of 4.16. This observation is compatible with the presence of magnetic contributions from both $\gamma$-Fe (fcc) and $\alpha-\mathrm{Fe}$ (bcc) at temperatures below $130 \mathrm{~K}$ [3638]. The diminution of the ZFC magnetisation at $130 \mathrm{~K}$ also suggests that the $\gamma$-Fe is in the antiferromagnetic state [21,24]. 

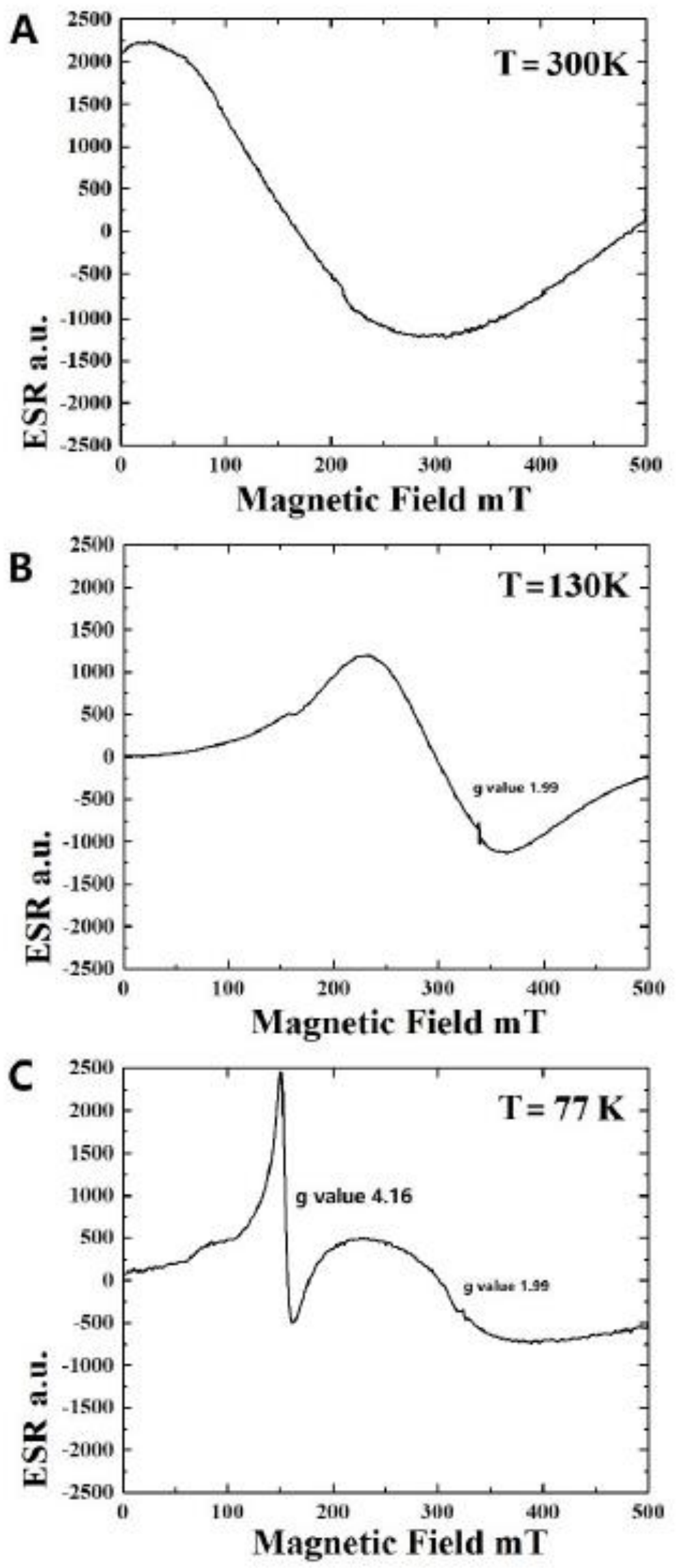

Figure 4: ESR spectra at (A) $300 \mathrm{~K}$, (B) $130 \mathrm{~K}$ and (C) $77 \mathrm{~K}$. 
DSC measurements show a decline in specific heat capacity in the temperature range at approximately $130 \mathrm{~K}$; this is further evidence for a significant structural transition at low temperature, Fig.5. The temperature dependence of the Seebeck coefficient, $S$, (Fig.6) is of the smoothly varying form generally observed in unmodified carbon nanotube networks, which comprises semiconductor- and metallike terms,

$S=\mathrm{A} T+(\mathrm{B}|\lambda| / T+\mathrm{C}) \exp (-\lambda / T)(1)$

where $A, B$, and $C$ are constants and $\lambda$ is the band-gap temperature of the semiconductor-like component [39]. The fitting of the data in Fig.6 to Equation 1 was achieved with A: $0.0257 \mu \mathrm{V} / \mathrm{K} 2, \mathrm{~B}:-0.0041 \mu \mathrm{V} / \mathrm{K}, \mathrm{C}:-20 \mu \mathrm{V} / \mathrm{K}, \lambda: 536 \mathrm{~K}$; the sign and magnitude of these values are comparable with those extracted from carbon nanotube networks $[39,40]$.

There are no large peaks associated with the Kondo effect in Fig.6 [41,42]; meaning there is no interaction of the magnetic moments of the filling with the spin of the conduction $\pi$-electrons in the graphitic carbon. 


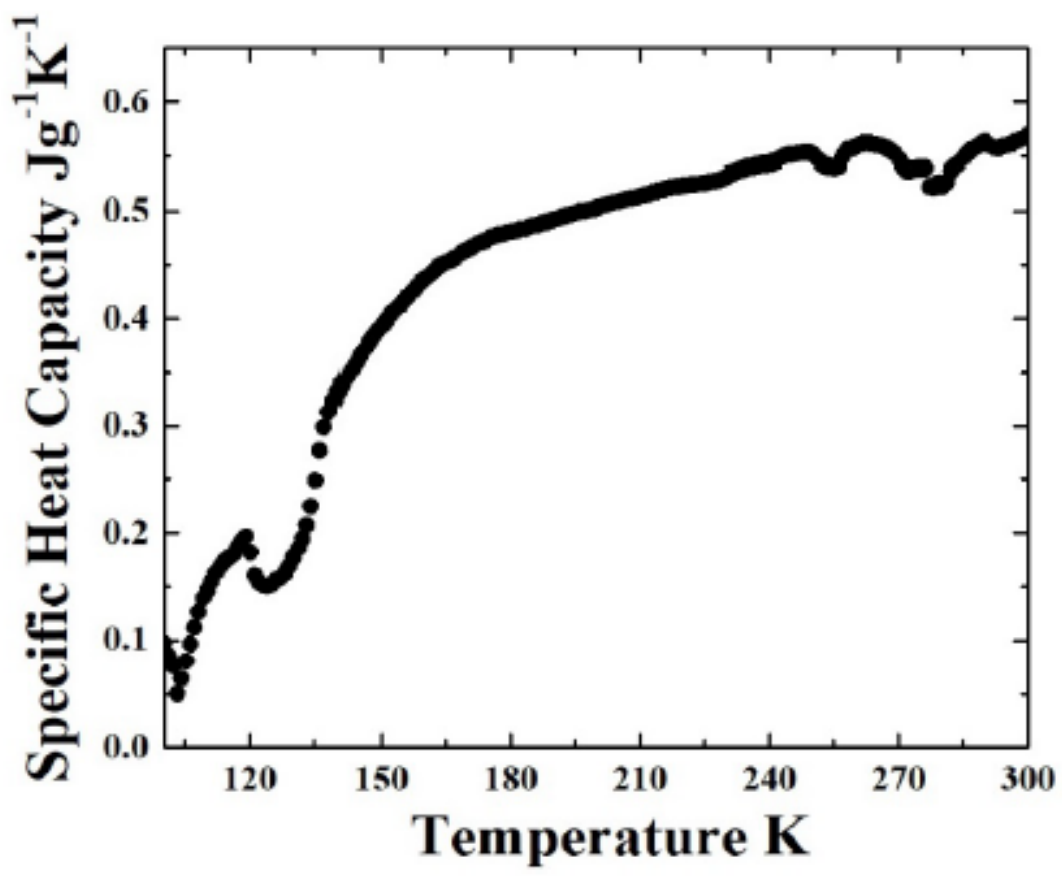

Figure 5: Specific heat capacity versus temperature obtained from the DSC measurement.



Figure 6: Variation of the Seebeck coefficient with the temperature. The solid line is the fit to Equation (1) with the values of parameters given in the text. 


\section{Conclusion}

In conclusion, iron-filled graphitic carbon foam exhibits ferromagnetic ordering at room temperature and both ferromagnetic and antiferromagnetic ordering at temperatures below $130 \mathrm{~K}$. There is no significant interaction between the magnetic moments in the iron and those due to the spin of $\pi$-electrons in the graphitic carbon.

\section{Acknowledgements}

We acknowledge the NSFC 11404227.

\section{Data Availability}

Additional characterization analyses can be found in the electronic supplementary information (ESI).

\section{References}

[1] Zhao X., Zhang Z., Wang L., Xi K., Cao Q., Wang D., Yang Y., Du Y. Excellent microwave absorption property of Graphene-coated Fe nanocomposites. Scientific Reports 2013; 3: 3421-5.

[2] Nikitenko S. I., Koltypin Y., Palchik O., Felner I., Xu X. N., Gedanken A. Synthesis of Highly Magnetic, Air-Stable Iron Carbide Nanocrystalline Particles by Using Power Ultrasound. Angew. Chem. Int. Ed. 2001; 40: 4447-9.

[3] Tang N.J., Chen W., Zhong W., Jiang H.Y., Huang S.L., Du Y.W. Highly stable carbon-coated $\mathrm{Fe} / \mathrm{SiO}_{2}$ composites: Synthesis, structure and magnetic properties. Carbon 2006; 44: 423-7. 
[4] Tang N.J., Zhong W., Wu X.L., Jiang H.Y., Liu W., Du Y.W. Synthesis and complex permeability of $\mathrm{Co} / \mathrm{SiO} 2$ nanocomposites. Materials Letters 2005; 59: 1723 6

[5] Lu X., Liang G., Zhang Y. Structure and magnetic properties of FeCo-SiO2 nanocomposite synthesized by a novel wet chemical method. Materials Letters 2007; 61: 4928-31.

[6] Wei X.J., Jiang J.T., Zhen L., Gong Y.X., Shao W.Z., Xu C.Y. Synthesis of $\mathrm{Fe} / \mathrm{SiO}_{2}$ composite particles and their superior electromagnetic properties in microwave band.

Materials Letters 2010; 64: 57-60.

[7] Cheng J., Ni X., Zheng H., Li B., Zhang X., Zhang D. Preparation of Fe (core)/SiO 2 (shell) composite particles with improved oxidation-resistance. Materials Research Bulletin 2006; 41: 1424-9.

[8] Tang N., Zhong W., Gedanken A., Du Y. A study of the stability of pyrolytic carbon-coated $\mathrm{Fe} / \mathrm{SiO}_{2}$ composites in $\mathrm{HNO}_{3}$ and the effect of pyrolysis temperatures on their magnetic properties. Solid State Communications 2007; 142: 265-9.

[9] Tang N.J., Jiang H.Y., Zhong W., Wu X. L., Zou W. Q., Du Y.W. Synthesis and magnetic properties of $\mathrm{Fe} / \mathrm{SiO} 2$ nanocomposites prepared by a sol-gel method combined with hydrogen reduction. Journal of Alloys and Compounds 2006; $419: 145-8$.

[10] Ni X., Zheng Z., Xiao X., Huang L., He L. Silica-coated iron nanoparticles: Shape controlled synthesis, magnetism and microwave absorption properties. Materials Chemistry and Physics 2010; 120: 206-12.

[11] Zhang X.F., Donga X.L., Huang H., Lv B., Zhu X.G., Lei J.P., Ma S., Liu W., 
Zhang Z.D. Synthesis, structure and magnetic properties of $\mathrm{SiO}_{2}$-coated $\mathrm{Fe}$ nanocapsules. Materials Science and Engineering A 2007; 454-455: 211-215. [12] Boi F. S., Guo J., Lan M., Yu T., Wang S., He Y., Wen J., Xiang G. Tuning high magnetizations in foam-like carbon-based films completely filled with $\alpha$-Fe. Carbon. Carbon, 2016; 101: 28-36.

[13] Zhang X., Wang S., He Y., Boi F. S. Mapping the transition from carbon-onions filled with $\mathrm{Fe}_{3} \mathrm{C}$ to carbon-foam completely filled with $\alpha-\mathrm{Fe}$ : Unlocking mass production of ferromagnetic carbon foam. Materials Today Communications 2018; 14: $72-76$.

[14] Zhang X., Medranda D., Odunmbaku O., Taallah A., and Boi F. S. Wustite induced formation of sp3-rich faceted carbon foam: the key role of cooling rate on sp3-abundance (and oxide crystal-habit) control. Materials Today Communications 2018; 16: 250-257.

[15] Boi F. S., Zhang X., Medranda D. Evidence of sp3-rich nano-diamond-like characteristics in amorphous carbon foam continuously filled with $\alpha$-Fe. Diamond and Related Materials 2018; 84: 190-195.

[16] Hudziak S., Darfeuille A., Zhang R., Peijs T., Mountjoy G., Bertoni G., et al. Magnetoresistive phenomena in an Fe-filled carbon nanotube/elastomer composite. Nanotechnology 2010; 21: 125505.

[17] Terrones H., Lopez-Urias F., Munoz-Sandoval E., Rodriguez-Manzo J. A., Zamudio A., Elias A.L. et al. Magnetism in Fe-based and carbon nanostructures: Theory and applications. Solid State Sciences, 2006; 8: 303-20. [18] Shpak A. P., Kolesnik S. P., Mogilny G. S., Petrov Yu. N., Sokhatsky V. P., Trophimova L. N., et al. Structure and magnetic properties of iron nanowires encased in multiwalled carbon nanotubes. Acta Materialia 2007; 55: 1769-78. 
[19] Dillon F. C., Bajpai A., Koos A., Downes S., Aslam Z. and Grobert N. Tuning the magnetic properties of iron-filled carbon nanotubes. Carbon 2012; 50: 3674-81.

[20] Hampel S., Leonhardt A., Selbmann D., Biedermann K, Elefant D., Muller Ch. et al. Growth and characterization of filled carbon nanotubes with ferromagnetic properties. Carbon 2006; 44: 2316-22.

[21] Prados C., Crespo P., Gonzalez J. M., Hernando A., Marco J. F., Gancedo R.,et al. Hysteresis shift in Fe-filled carbon nanotubes due to $\gamma$-Fe. Physical Review B 2002; 65: 113405.

[22] Shi C. X., Cong H. T. Tuning the coercivity of Fe-filled carbon-nanotube arrays by changing the shape anisotropy of the encapsulated Fe nanoparticles. Journal of Applied Physics 2008; 104: 034307.

[23] Leonhardt A., Hampel S., Muller C., Monch I., Koseva R., Ritschel M., et al. Synthesis, Properties, and Applications of Ferromagnetic-Filled Carbon Nanotubes. Chemical Vapor Deposition 2006; 12: 380-7.

[24] Karmakar S., Sharma S. M., Mukadam M. D., Yusuf S. M. and Sood A. K. Magnetic behaviour of iron-filled multiwalled carbon nanotubes. Journal of Applied Physics 2005; 97: 054306.

[25] Weissker U, Hampel S, Leonhardt A, Buchner B. Carbon Nanotubes Filled with Ferromagnetic Materials. Materials 2010; 3: 4387-427.

[26] Lv R., Tsuge S., Gui X., Takai K., Kang F., Enoki T. et al. In situ synthesis and magnetic anisotropy of ferromagnetic buckypaper. Carbon 2009; 47: 1141.

[27] Boi F. S., Mountjoy G., Wilson R. M., Luklinska Z., Sawiak L. J. and Baxendale M. Multiwall carbon nanotubes continuously filled with micrometre-length ferromagnetic $\alpha$-Fe nanowires. Carbon 2013; 64: 351-8.

[28] F. S. Boi F. S., Mountjoy G., Baxendale M. Boundary layer chemical vapor 
synthesis of self-organized radial filled-carbon-nanotube structures. Carbon 2013; 64:516-26.

[29] Jiwei Li, Jianwei Ma, Shaojuan Chen, Jinmei He, Yudong Huang.

Characterization of calcium alginate/ deacetylated konjac glucomannan blend films prepared by Ca2p crosslinking and deacetylation. Food Hydrocolloids 2018; 82: 363369.

[30] Guanglei Wu, Hongxia Zhang, Xixi Luo, Lieji Yang, Hualiang Lv. Investigation and optimization of $\mathrm{Fe} / \mathrm{ZnFe} 2 \mathrm{O} 4$ as a Wide-band electromagnetic absorber. Journal of Colloid and Interface Science 2019; 536: 548-555.

[31] Guanglei Wu, Zirui Jia, Yonghong Cheng, Hongxia Zhang, Xinfeng Zhou, Hongjing Wu. Easy synthesis of multi-shelled $\mathrm{ZnO}$ hollow spheres and their conversion into hedgehog-like $\mathrm{ZnO}$ hollow spheres with superior rate performance for lithium ion batteries. Applied Surface Science 2019; 464: 472-478.

[32] Jiwei Li, Jianwei Ma, Shaojuan Chen, Yudong Huang, Jinmei He. Adsorption of lysozyme by alginate/graphene oxide composite beads with enhanced stability and mechanical property. Materials Science \& Engineering C 2018; 89: 25-32. [33] Guanglei Wu, Yonghong Cheng, Zhihong Yang, Zirui Jia, Hongjing Wu, Lieji Yang, Hongliang Li, Peizhi Guo, Hualiang Lv. Design of carbon sphere/magnetic quantum dots with tunable phase compositions and boost dielectric loss behavior. Chemical Engineering Journal 2018; 333: 519-528.

[34] Mingliang Ma, Yuying Yang, Dili Liao, Ping Lyu, Jinwei Zhang, Jianli Liang, Lizhi Zhang. Synthesis, characterization and catalytic performance of core - shell structure magnetic $\mathrm{Fe}_{3} \mathrm{O}_{4} / \mathrm{P}(\mathrm{GMA}-\mathrm{EGDMA})-\mathrm{NH}_{2} / \mathrm{HPG}-\mathrm{COOH}-\mathrm{Pd}$ catalyst. Appl. Organometal. Chem. 2019; 33: e4708. 
[35] Mingliang Ma, Yuying Yang, Wenting Li, Renjun Feng, Zhangwen Li, Ping Lyu, and Yong Ma. Gold nanoparticles supported by amino groups on the surface of magnetite microspheres for the catalytic reduction of 4-nitrophenol. J Mater Sci 2019; 54: 323-334.

[36] Sohatsky V., Kolesnik S., Makarov D., Leonhardt A., Muehl T., Moench I., Ritschel M., Kozhuharova R. K., Schumann J. and Schneider C. M. ESR of Fe-Filled Multi-Walled Carbon Nanotubes. Fullerenes, Nanotubes and Carbon Nanostructures 2005; 13: 401-410.

[37] Dresselhaus, M.S., Dresselhaus, G., and Avouris, Ph. (2001) Carbon Nanotubes: Synthesis, Structure, and Applications; Springer-Verlag: New York. [38] Hihara T., Onodera H., Sumiyama K., Suzuki K., Kasuya A., Nishina Y., Saito Y., Yoshikawa T. and Okuda M. Magnetic properties of iron in nanocapsules. Japanese Journal of Applied Physics 1994; 33:24-25.

[39] Hone J., Ellwood I., Muno M., Mizel A., Cohen M. L. and Zettl A., Rinzler A. G. and Smalley R. E. Thermoelectric Power of Single-Walled Carbon Nanotubes. Phys. Rev. Lett. 1998; 80: 1042-1045.

[40] Grigorian L., Sumanasekera G. U., Loper A. L., Fang S. L., Allen J. L., and Eklund,P. C. Giant thermopower in carbon nanotubes: A one-dimensional Kondo system. Phys Rev. B 1999; 60: R11 309-312.

[41] Sun P., Wei B., Zhang J., Tomczak J. M., Strydom A. M., Søndergaard M., Iversen B. B. and Steglich F. Large Seebeck effect by charge-mobility engineering. Nature Communications 2015; 6: 7475.

[42] Zitko R., Mravlje J., Ramsak A. and T. Rejec. Spin thermopower in the overscreened Kondo model. New Journal of Physics 2013;15:105023 
[43] Eckmann A., Felten A., Mishchenko A., Britnell L., Krupke R., Novoselov K. S. and Casiraghi C. Probing the Nature of Defects in Graphene by Raman Spectroscopy. Nano Lett. 2012; 12: 3925-3930.

[44] Sheng Z.M., Wang J.N., Growth of magnetic carbon with a nanoporous and graphitic structure, Carbon 2009; 47: 3271-3279.

[45] Barros E.B., Demir N.S., Souza Filho A.G., Mendes Filho J., Jorio A., Dresselhaus G., et al., Raman spectroscopy of graphitic foams, Phys. Rev. B 2005; 71: 165422. 